\title{
Anaerobic Biotransformation of Nitro-Compounds to Amines by Bovine Rumen Fluid
}

\author{
Alfonso Rodríguez ${ }^{1}$, Ignacio Irazoqui ${ }^{1}$, Guzmán Álvarez ${ }^{1}$, Cecilia Cajarville ${ }^{2}$, José Luis Repetto ${ }^{3}$, \\ Mercedes González ${ }^{1}$, Hugo Cerecetto ${ }^{1 *}$ \\ ${ }^{1}$ Grupo de Química Medicinal-Laboratorio de Química Orgánica, Facultad de Ciencias-Facultad de Química, \\ Universidad de la República, Iguá, Montevideo, Uruguay \\ ${ }^{2}$ Departamento de Nutrición, Facultad de Veterinaria, Universidad de la República, Lasplaces, Montevideo, Uruguay \\ ${ }^{3}$ Departamento de Bovinos, Facultad de Veterinaria, Universidad de la República, Lasplaces, Montevideo, Uruguay \\ E-mail: "hcerecet@fq.edu.uy,hcerecetto@gmail.com \\ Received March 17, 2011; revised April 25, 2011; accepted May 9, 2011
}

\begin{abstract}
Microorganims of the bovine rumen fluid biocatalyzed the reduction of nitro-compound substrates to yield the respective amines. This enzymatic process, using ruminal contents, has rarely been reported in association with the bioreduction of nitro groups. The biotransformation reactions catalyzed by this system were dependent of both the electronic characteristics and the area/volume of the nitro-substrates confirming the processes are enzymatic. The semi-preparative scale biotransformation went by in good yield showing the rumen fluid may be employed in the synthesis of amines under very mild conditions and, moreover, it may have application in the bioremediation of nitro-compounds.
\end{abstract}

Keywords: Biotransformation, Nitro-Compounds, Amines, Rumen, Ruminal Fluid, Bovine

\section{Introduction}

Much work has been done to find effective ways of bioremediating of serious environmental nitro-substituted contaminants, i.e. the explosives TNT, HMX, and RDX [1-5]. Efforts have focused on a variety of living organisms and microbes containing ecosystems able to reduce the nitro group to amine, hydroxylamine, or other intermediates like nitroso and azoxy compounds [6,7]. On the other hand, many chemicals and pharmaceuticals are or require an amine intermediates and only relatively small number of chemical routes have involve safety, scalability and environmental acceptability for large-scale manufacturing [8-10]. In this sense, several biocatalytic processes have been also described $[7,11,12]$.

The digestive anatomy and physiology of cow and other ruminants is markedly different to that of monogastric animals such as man. The ruminants have three additional compartments (rumen, reticulum and omasum) before the true stomach (abomasum). These compartments allow the microbial population to extract and the host to absorb energy from fibrous plant material not otherwise available to mammalian enzymes. Digestion of food in the rumen occurs by a combination of microbial fermentation and physical breakdown during rumination being the rumen a highly reductive anaerobic environment [13]. The products of fermentation are mainly the acetate, propionate, butyrate, formate, ethanol, lactate, succinate, ammonia, carbon dioxide and hydrogen gas [14]. The total pool of hydrogen gas is produced during microbial fermentation of feed, and is used as an energy source by methanogenic archaea producing methane. This pool is small and the dissolved $\mathrm{H}_{2}$ concentration is usually about $0.1-50 \mu \mathrm{m}$, which is $0.014 \%$ to $6.8 \%$ of its maximal solubility at $39^{\circ} \mathrm{C}$ and one atmosphere pressure. Efficient $\mathrm{H}_{2}$ removal is postulated to increase the rate of fermentation by eliminating the inhibitory effect of $\mathrm{H}_{2}$ on the microbial degradation of plant material. Anaerobic microbial community in the rumen which consists of many bacterial, archaeal, protozoal and fungal species, could be able to biotransform xenobiotics to new compound. Examples of xenobiotic transformations by rumen microbes have been reported for toxicants in forages such as pyrrolizine alkaloids, fumonisin, alflatoxin [15-18], and nitropropionic acid, and TNT that were converted to the corresponding amine and hydroxylamine derivatives $[4,19]$.

This study looked at the capacity of bovine ruminal 
microorganisms to biotransform nitro-compounds under anaerobic conditions. The present paper describes the reductive potential of cow rumen with respect to six nitroaromatic and two nitro-arenic substrates (Scheme 1) containing different groups connected to the nitro-moiety in order to establish the structural requirements for optimal biotransformation. Apart from the noticeable electronic effect of some of the substituent, electron-withdrawing and electron-donor moieties (1-3 and 4-6, respectively), we selected them for their ability to suffer potentially other biotransformations, hydrolysis $\left(-\mathrm{CO}_{2} \mathrm{Et}\right)$, reduction $\left(-\mathrm{CO}_{2} \mathrm{Et}\right.$, $-\mathrm{COCH}_{3}$, $\left.-\mathrm{CH}=\mathrm{CH}\right)$, oxidation $\left(-\mathrm{CH}_{3}\right)$, aldol reaction $\left(-\mathrm{COCH}_{3}\right)$, and consequently to evidence whether the bovine rumen fluid is able to perform these processes.

\section{Materials and Methods}

\subsection{General Methods}

Products of biotransformations were purified by chromatography column $\left(\mathrm{SiO}_{2}, 230\right.$ - 400 mesh; eluted with mixtures of EtOAC:petroleum ether, 9:1; 8:2, and 1:1). The purification and progresses of the biotransformations were checked by TLC (silica gel 60 F254 layers, EtOAc: petroleum ether, 1:1), visualised under UV light $(\lambda=254$ $\mathrm{nm}$ ), by exposing to iodine vapor, by spraying with $p$ anisaldehyde/sulphuric acid reagent and heating at ca. $120^{\circ} \mathrm{C}$, or by spraying with 2,4-dinitrophenylhydrazine/ sulphuric acid reagent. The identities of products were determined using their chromatographic characteristics and ${ }^{1} \mathrm{H}$ NMR spectra by comparison with those of reference standards. ${ }^{1} \mathrm{H}$ NMR spectra were measured at 400 $\mathrm{MHz}$ using a Bruker DPX400 instrument with the analyte dissolved in $\mathrm{CDCl}_{3}$.

\subsection{Chemicals}

The reagents, the nitro-compounds 1-6, and the amines 1a-8a and 3b (Scheme 1) were obtained in analytical grade from Sigma-Aldrich and Merck and were used without further purification. The nitro-compounds 7 and 8 were prepared as previously [20].

\subsection{Standard Procedure for Small Scale Biotransformation Reactions}

Microbial sources and collection: Rumen fluid was collected from a cannulated dairy cow ( $550 \mathrm{~kg}$ body weight), fed a $70 \%$ forage and $30 \%$ concentrate diet at an intake level of $3 \%$ of the body weight. The procedure was approved by the Bioethics Committee of Veterinary Faculty (UdelaR). The collection of rumen fluid was performed approximately $1-2 \mathrm{~h}$ after the beginning of the main meal and filtered through two layers of cheesecloth into $1 \mathrm{~L}$ plastic prewarmed $\left(39^{\circ} \mathrm{C}\right)$ vessel with no remaining air space and under $\mathrm{CO}_{2}$ atmosphere. The vessels were sealed and transported to the laboratory within $1 \mathrm{~h}$. The ruminal fluid was maintained no more a week at $4^{\circ} \mathrm{C}$.<smiles>[R]c1ccc([N+](=O)[O-])cc1</smiles>
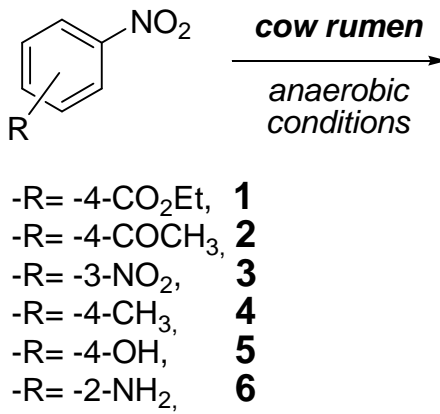<smiles>[R]c1ccc(N)cc1</smiles>

$-\mathrm{R}=-4-\mathrm{CO}_{2} \mathrm{Et}, \quad \mathbf{1} \mathbf{a}$

$-\mathrm{R}=-4-\mathrm{COCH}_{3}, \mathbf{2 a}$

$-\mathrm{R}=-3-\mathrm{NO}_{2}, \quad \mathbf{3 a} \longrightarrow-\mathrm{R}=3-\mathrm{NH}_{2}, \mathbf{3 b}$

$-\mathrm{R}=-4-\mathrm{CH}_{3}$

$-\mathrm{R}=-4-\mathrm{OH}$,

$-\mathrm{R}=-2-\mathrm{NH}_{2}$,

$5 a$

$6 a$
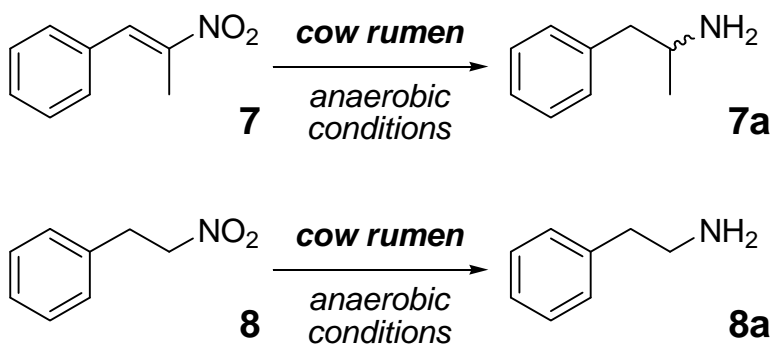

Scheme 1. Nitro-compounds 1-8 employed as substrates in biotransformation studies involving bovine rumen fluid and expected generated products (1a-8a, and $3 b)$. 
Culture conditions and sampling protocols: Transformation experiments were conducted under anaerobic conditions in $10 \mathrm{~mL}$ vials containing $1 \mathrm{~mL}$ of the rumen fluid and $4 \mathrm{~mL}$ of McDougall's buffer [21]. The nitrocompounds dissolved in methanol were added to a final concentration of $200 \mathrm{mg} / \mathrm{L}$. The vials were purged with $\mathrm{N}_{2}$ prior to being sealed with white rubber serum stoppers and plastic crimp closures. The vials were incubated at $39^{\circ} \mathrm{C}$, protected from light, without agitation. Samples were withdrawn from duplicate vials at the appropriate time intervals $(0.5,1,4,6$, and $24 \mathrm{~h})$ and analyzed immediately for nitro-compounds and metabolic products. Samples were treated with methanol (500 $\mu \mathrm{L} / 1 \mathrm{~mL}$ of rumen) and stirred during $30 \mathrm{~min}$ in order to destroy the biological matrix. Then EtOAc $(3 \mathrm{~mL})$ and saturated aqueous $\mathrm{NaHCO}_{3}$ solution $(3 \mathrm{~mL})$ were added and the organic layer was evaporated in vacuo to be analyzed by chromatography. Metabolite identification was based on comparison of chromatographic behaviors. Samples were analyzed immediately following collection to minimize abiotic degradation of intermediates. Different controls were used to confirm the origin of the biotransformation: a) autoclaved opened-rumen fluid/McDougall's buffer mixture and nitro-compounds; b) $\gamma$-irradiated sealed-rumen fluid/McDougall's buffer mixture and nitro-compounds; c) continuous nitrogen-bubbled rumen fluid/McDougall's buffer mixture and nitro-compounds; d) hydrogen saturated McDougall's buffer and nitro-compounds; e) McDougall's buffer and nitro-compounds; and f) rumen fluid/McDougall's buffer mixture.

\subsection{Procedure for the Scale-up Biotransformation}

Semi-preparative protocol was performed with nitrocompound 2 (30 mg in $100 \mathrm{~mL}$ of ruminal fluid, $400 \mathrm{~mL}$ of McDougall's buffer, and $5 \mathrm{~mL}$ of $\mathrm{MeOH}$ ) incubated during 5 days in the same conditions that it is indicated in Section 2.3. After the incubation time the bio-material was treated as it is indicated in Section 2.3. The residue from in vacuo evaporation was purified by chromatographic column as it is indicated in Section 2.1., the identity of the main product was confirmed by ${ }^{1} \mathrm{H}$ NMR spectrum.

\subsection{Theoretical Calculations}

The molecular structures of nitro-compounds, 1-8, and the corresponding products of bioreduction, 1a-7a, were subjected to complete geometry optimization, in gas phase, using PC SPARTAN 04 package [22] as follows: conformational search using MMFF conformer module, after that from the most stable conformer fully optimi- zation by applying Hartree-Fock 6-31G*. Structural, geometrical, electronic and hydrophobic descriptors were extracted from the calculus.

\section{Results and Discussion}

Biotransformations of the nitro-compounds 1-8 (Scheme 1) were carried out using bovine rumen fluid obtained from dairy cows. The processes were conducted either on a small scale (1 mg of substrate solubilised in methanol in $1 \mathrm{~mL}$ of ruminal fluid and $4 \mathrm{~mL}$ of McDougall's buffer, which is similar to the bicarbonate buffer from the salivary glands of the ruminant [23]) or on a semipreparative scale $(500 \mathrm{~mL}$ of ruminal fluid/McDougall's buffer, $30 \mathrm{mg}$ of nitro-compound 2 solubilised in methanol) and the mixtures were incubated without agitation for 24 hours, in the small scale, or 5 days, in semi-preparative scale, at $39^{\circ} \mathrm{C}$. Results of small scale bioreduction are presented in Table 1. The small scale biotransformations were checked at different times $(0.5,1,4,6$, and $24 \mathrm{~h}$ ) being nitro-compound 1 biotransformed by ruminal fluid to the corresponding amine (1a) since $0.5 \mathrm{~h}$ carrying out a complete reduction at $1 \mathrm{~h}$ of incubation. The same behavior was observed for the nitro-alkane 8 which was completely biotransformed to the propylamine derivative 8a and to a second product which was not structurally identified. Nitro-compounds $\mathbf{2}$ and $\mathbf{3}$ were also converted into the corresponding amines, 2a and 3a, almost completely at $24 \mathrm{~h}$ of incubations. No formation of amines were evidenced at short times of incubation for substrates 2, and 4-7, however lower amounts of the reduction products were formed with substrates $\mathbf{4}$, and 5 after $24 \mathrm{~h}$ of incubation. No detectable amine-product was observed with nitro-compound $\mathbf{6}$.

In order to confirm the biological origin of the reduction some experiments were performed using the $1 \mathrm{~h}$ most reactive nitro-compound, derivative 1 (Table 2). The absence of reduction product, $\mathbf{1 a}$, from the inactivated rumen (control a) and from the chemical conditions (control d, and control e) indicated that the transformations involved biological systems. The presence of traces of 1a, from $\mathbf{1}$, in control b (inactivated rumen fluid by $\gamma$-irradiated in sealed-vial and with the initial hydrogen atmosphere) and control c (normal incubation but with continuous nitrogen-bubbled flow) pointed to the presence of hydrogen, from the ruminal microbial fermentation, is also important for adequate biotransformation. The capability of ruminal fluid to promote others biotransformations was analyzed checking the presence of potential side products, 9-14. Scheme 2 summarizes the undetected studied subproducts. Presence of carbonyl compound 12 and 13 was analyzed due to this biotransformation, $\mathbf{7}$ to $\mathbf{1 2}$ or $\mathbf{8}$ to $\mathbf{1 3}$, was previously described 
Table 1. Small scale biotransformations of nitro-compounds by bovine rumen fluid.

\begin{tabular}{ccc}
\hline \multirow{2}{*}{ Nitro-compound } & \multicolumn{2}{c}{ Percentage of biotransformation $(\%)^{\mathrm{a}}$} \\
\cline { 2 - 3 } & 1 h of incubation & 24 h of incubation $^{\mathrm{b}}$ \\
\hline $\mathbf{1}$ & $100^{\mathrm{b}}$ & 100 \\
$\mathbf{2}$ & 0 & 40 \\
$\mathbf{3}$ & 90 & $100^{\mathrm{c}}$ \\
$\mathbf{4}$ & 0 & 10 \\
$\mathbf{5}$ & 0 & 0 \\
$\mathbf{6}$ & 0 & $0^{\mathrm{d}}$ \\
$\mathbf{7}$ & 0 & $\mathrm{~ns}^{\mathrm{e}}$ \\
$\mathbf{8}$ & 100 & 100 \\
\hline
\end{tabular}

${ }^{\mathrm{a}}$ The analyzed products were 1a-8a, and $\mathbf{3 b}$. ${ }^{\mathrm{b}}$ The same results were obtained in absence of McDougall's buffer. ${ }^{\mathrm{c}} 100 \%$ of biotransformation to 3a, amino-derivative $3 \mathbf{b}$ was not detected at any of the studied times. ${ }^{\mathrm{d}}$ After 48 $\mathrm{h}$ of incubation product $\mathbf{6 a}$ was also not detected. ${ }^{\mathrm{e}}$ ns: not studied.

Table 2. Results of the biotransformations of nitro-compound 1 to amine derivative 1a by incubation during $1 \mathrm{~h}$ in different conditions.

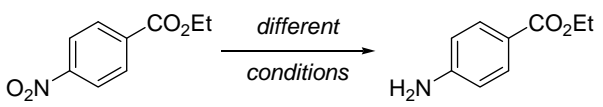

\begin{tabular}{ccccccccc}
\multicolumn{1}{c}{1} & \multicolumn{1}{c}{ Conditions $^{\mathrm{a}}$} \\
\hline & normal & $c^{\mathrm{b}} a$ & $c b$ & $c c$ & $c d$ & $c e$ & $c f$ \\
\hline $\begin{array}{c}\text { presence of } \\
\text { amine 1a }\end{array}$ & $100 \%{ }^{\mathrm{c}}$ & $\mathrm{abs}^{\mathrm{d}}$ & traces & traces & abs & abs & abs \\
\hline
\end{tabular}

${ }^{a}$ For details see Materials and methods (section 2.3). ${ }^{b}$ : control. ${ }^{c}$ See Table 1. ${ }^{\mathrm{d}}$ abs: absence.

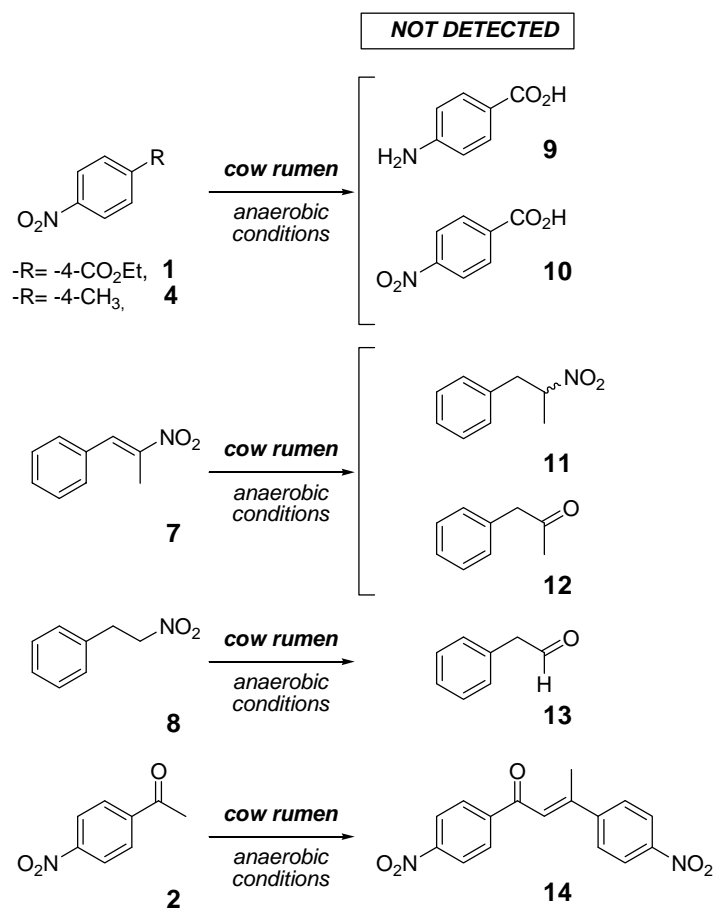

Scheme 2. Undetected side processes. using whole cells of Peptostreptococcus productus U-1 [24]. Finally, during the biotransformation of nitro-compound 2 some by product was detected which was not structurally identified. However, according to the chromatographic behavior and the consumption during the time it could be hypothesized this compound is the corresponding hydroxylamino-intermediate.

The semi-preparative scale biotransformation of nitrocompound 2 produced the corresponding amine product 2a in a $60 \%$ of yield after chromatographic isolation. The product was characterized by ${ }^{1} \mathrm{H}$ NMR (see Supplementary Material).

It was described that the bioreduction of the nitroaromatic substrate, by other organisms [25], is dependent of the electronic characteristic of the rest of the aromaticsubstituents. Herein, it is in general true. For example the nitro-compounds $\mathbf{1}$ and $\mathbf{3}$, substituted by electron-withdrawing moieties, were reduced almost completely after $1 \mathrm{~h}$ of incubation with ruminal fluid, and nitro-compounds 5, 6, and product 3a, substituted by electrondonor moieties, remained inalterable after $24 \mathrm{~h}$ of incubation. The electron-withdrawing substituents decrease the electron deficiency of the nitro-aromatic ring and, consequently, a lower redox potential is required to reduce the nitro group. This effect was clearly visualized in the LUMO maps of nitro-reactants and in the SHOMO maps of the nitro-anion radical intermediates (Scheme 3(a)) where the best-bioreduced derivatives, i.e. 1, 2, and 8, posses similar maps of the lowest unoccupied molecular orbitals. However, the acetyl-substituted derivative, nitro-compound 2, was not biotransformed after $1 \mathrm{~h}$ of incubation and only $40 \%$ was converted into the aminoderivative $2 \mathrm{a}$ after $24 \mathrm{~h}$ of bioreduction. In the semipreparative protocol it was necessary to incubate 5 days to allow the complete transformation. In order to explain the unexpected behavior of compound 2 we analyzed the processes in theoretical terms trying to identify the relationship between structure and biotransformation results. For this, molecular modeling studies were performed on the nitro-compounds 1-6, 8, and 3a determining physicochemical descriptors potentially related to bioreduction (see Supplementary Material). After that, relationships between percentage of biotransformations at $24 \mathrm{~h}$ (PB), dependent variable, and the different calculated descriptors, independent variables, were analyzed. For nitroaromatic compounds, 1-6, and 3a, statistical significant correlation was obtained when the energy difference, products (amino-compounds) - reactive (nitro-compound) $(\Delta \mathrm{E})$, was used as independent variable (Scheme 3(b)). However, nitro-aliphatic $\mathbf{8}$ did not match in this correlation. Consequently, multivariable regressions between the PB and the physicochemical descriptors were studied. When the complete compound-population was studied 


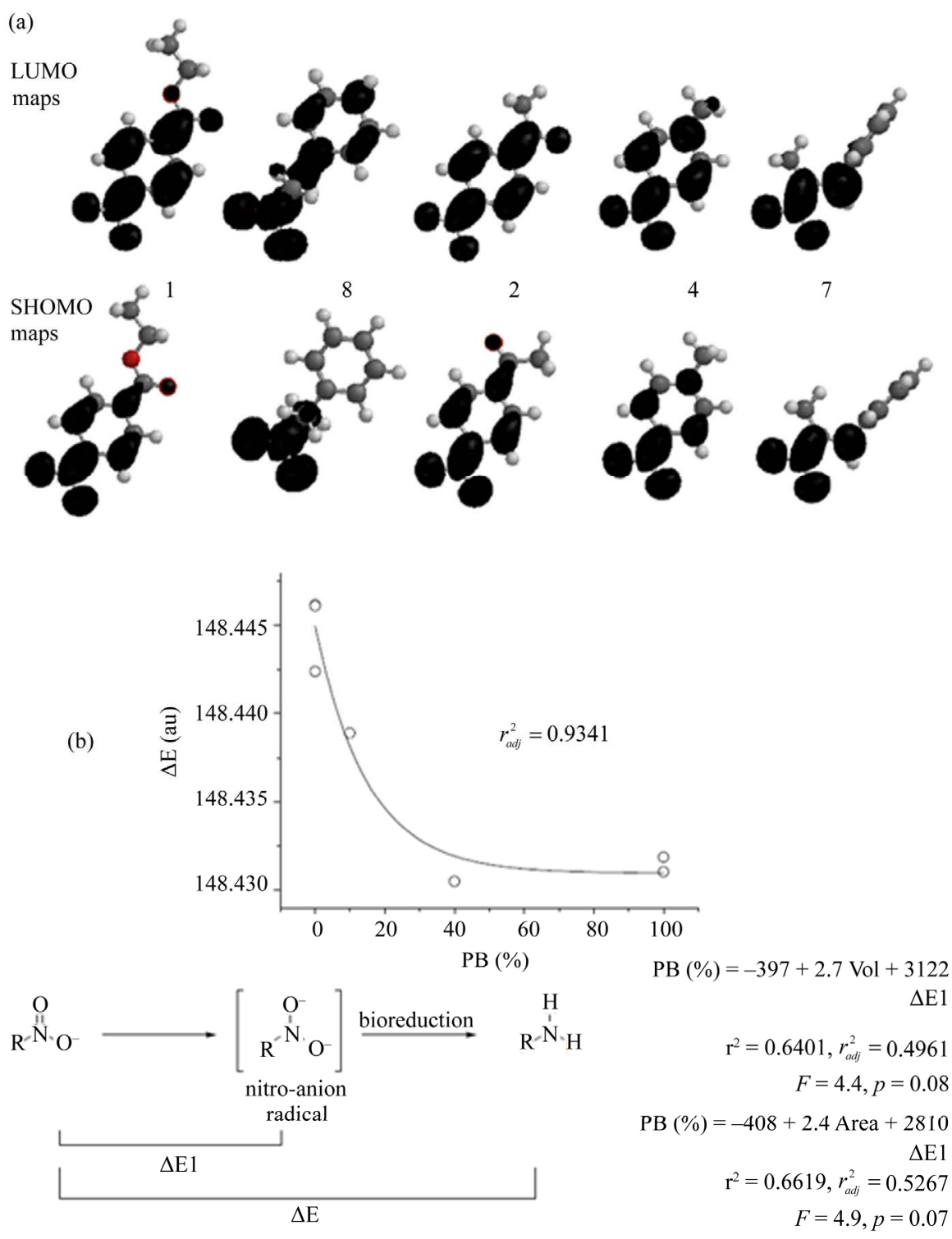

(c)

Scheme 3. (a) Maps of the LUMO, for the nitro-reactant, and SHOMO, for the nitro-anion radical. (b) and (c) Relationships between percentage of amino products at $24 \mathrm{~h}$ incubation (PAP) and studied physicochemical descriptors. (b) Relationship from nitro-aromatic substrates (1-6, and 3a). (c) Relationship from studied nitro-compounds (1-6, 8, and 3a).

adequate correlations were found between PB and energy difference between the nitro-anion radical intermediate and the nitro-reactive $(\Delta \mathrm{E} 1)$, and area of the nitrocompounds $\left(r^{2}=0.6619, r_{a d j}^{2}=0.5267\right.$, Scheme 3(c) $)$ or between $\mathrm{PB}$ and $\Delta \mathrm{E} 1$, and volume of the nitro-compounds $\left(r^{2}=0.6401, r_{a d j}^{2}=0.4961\right.$, Scheme 3(c)). As one expected, these correlations showed the relevance of the energies of the processes in the appearance of the amine products, and the area/volume of the nitro-compounds showing that an enzymatic process could be involved due to the size of the compounds play a role in the amine production.

\section{Conclusions}

Bovine rumen fluid has been shown to be efficient in the biotransformation of a number of nitro-substrates, producing amines in good concentrations. It is necessary to point out that this study was performed using rumen fluid from a cow with a pre-established nutritional regime, conserving the fluid at a low temperature, during several days. Consequently, the results could be modified if the fluid was immediately used or if it was preserved under physiological conditions [26]. Additionally, the type and the management of the diet received by the fluid donors 
are factors that strongly influence rumen fluid microbiota [27]. All these aspects should be considered for future research.

The results suggest that ruminal fluid may offer some application in the synthesis of amines, under very mild conditions, and in the bioremediation of toxic nitrocompounds involving safety, scalability and environmentally friendly conditions.

\section{Acknowledgements}

We thank Dr. Pablo Cabral, from Centro de Investigaciones Nucleares-Udelar, for the $\gamma$-irradiation of rumen.

\section{References}

[1] A. Esteve-Núñez, A. Caballero and J. L. Ramos, "Biological Degradation of 2,4,6-Trinitrotoluene," Microbiology and Molecular Biology Reviews, Vol. 65, No. 3, 2001, pp. 335-352. doi:10.1128/MMBR.65.3.335-352.2001

[2] Z. Snellinx, A. Nepovírm, S. Taghavi, J. Vangronsveld, T. Vanek and D. van der Lelie, "Biological Remediation of Explosives and Related Nitroaromatic Compounds," Environmental Science Pollution Research International, Vol. 9, No. 1, 2002, pp. 48-61. doi:10.1007/BF02987316

[3] J. L. Ramos, M. M. González-Pérez, A. Caballero and P. van Dillewijn, "Bioremediation of Polynitrated Aromatic Compounds: Plants and Microbes Put up a Flight," Current Opinion in Biotechnology, Vol. 16, No. 3, 2005, pp. 275-281. doi:10.1016/j.copbio.2005.03.010

[4] T. J. Fleischmann, K. C. Walker, J. C. Spain, J. B. Hughes and A. M. Craig, "Anaerobic Transformation of 2,4,6TNT by Bovine Ruminal Microbes," Biochemical and Biophysical Research Communications, Vol. 314, No. 4, 2004, pp. 957-963. doi:10.1016/j.bbrc.2003.12.193

[5] E. L. Rylott, R. G. Jackson, F. Sabbadin, H. M. B. SethSmith, J. Edwards, C. S. Chong CS, S. E. Strand, G. Grogan and N. C. Bruce, "The Explosive-Degrading Cytochrome P450 XplA: Biochemistry, Structural Features and Prospects for Bioremediation," Biochimica et Biophysica Acta, Vol. 1814, No. 1, 2011, pp. 230-236.

[6] K.-H. Blotevogel and T. Gorontzy, "Microbial Degradation of Compounds with Nitro Functions," In: J. Klein, Ed., Biotechnology, Vol. 11b: Environmental Processes II, Wiley-VCH, Weinheim, 2000, pp. 274-302.

[7] M. Kulkarni and A. Chaudhari, "Microbial Remediation of Nitro-Aromatic Compounds: An Overview," Journal of Environmental Management, Vol. 85, No. 2, 2007, pp. 496-512. doi:10.1016/j.jenvman.2007.06.009

[8] J.M. Brunel and G. Buono, "Enantioselective Borane Catalyzed Reduction of Imines," Synlett, Vol. 2, 1996, pp. 177-178. doi:10.1055/s-1996-5343

[9] L. Pehlivan, E. Métay, S. Laval, W. Dayoub, P. Demonchaux, G. Mignani and M. Lemaire, "Alternative Method for the Reduction of Aromatic Nitro to Amine Using TMDS-Iron Catalyst System," Tetrahedron, Vol. 67, No.
10, 2011, pp. 1971-1976. doi:10.1016/j.tet.2010.12.070

[10] W. Peng, F. Zhang, G. Zhang, B. Liu and X. Fan, "Selective Reduction of 4,4'-Dinitrostilbene-2,2'-Disulfonic Acid Catalyzed by Supported Nano-Sized Gold with Sodium Formate as Hydrogen Source," Catalysis Communications, Vol. 12, No. 6, 2011, pp. 568-572. doi:10.1016/j.catcom.2010.12.006

[11] D. E. Gibbs and D. Barnes, "Asymmetric Synthesis of Amines by Action of Baker's Yeast on Oximes," Tetrahedron Letters, Vol. 31, No. 39, 1990, pp. 5555-5558.

[12] J. A. Blackie, N. Turner and A. Wells, "Concerning the Baker's Yeast (Saccharomyces cerevisiae) Mediated Reduction of Nitroarenes and Other N-O Containing Functional Groups," Tetrahedron Letters, Vol. 38, No. 17, 1997, pp. 3043-3046. doi:10.1016/S0040-4039(97)00504-2

[13] D. N. Kamra, "Rumen Microbial Ecosystem," Current Science, Vol. 89, No. 1, 2005, pp. 124-135.

[14] P. H. Janssen, "Influence of Hydrogen on Rumen Methane Formation and Fermentation Balances through Microbial Growth Kinetics and Fermentation Thermodynamics," Animal Feed Science and Technology, Vol. 160, No. 1-2, 2010, pp. 1-22. doi:10.1016/j.anifeedsci.2010.07.002

[15] J. T. Hovermale and A. M. Craig, "Metabolism of Pyrrolizidine Alkaloids by Peptostreptococcus Heliotrinreducens and a Mixed Culture Derived from Ovine Ruminal Fluid," Biophysical Chemistry, Vol. 101-102, No. 10, 2002, pp. 387-399. doi:10.1016/S0301-4622(02)00152-7

[16] N. K. Gurung, D. L. Rankins and R. A. Shelby, "In Vitro Ruminal Disappearance of Fumonisin B1 and Its Effects on in Vitro Dry Matter Disappearance," Veterinary \& Human Toxicology, Vol. 41, No. 4, 1999, pp. 196-199.

[17] R. Hedman and H. Pettersson, "Transformation of Nivalenol by Gastrointestinal Microbes," Archiv fur Tierernahrung, Vol. 50, No. 4, 1997, pp. 321-329.

[18] K. H. Kiessling, H. Pettersson, K. Sandholm and M. O1sen, "Metabolism of Aflatoxin, Ochratoxin, Zearalenone and Three Trichothecenes by Intact Rumen Fluid, Rumen Protozoa, and Rumen Bacteria," Applied and Environmental Microbiology, Vol. 47, No. 5, 1984, pp. 10701073.

[19] R. C. Anderson, M. A. Rasmussen and M. J. Allison, "Metabolism of the Plant Toxins Nitropropionic Acid and Nitropropanol by Ruminal Microorganisms," Applied and Environmental Microbiology, Vol. 59, No. 9, 1993, pp. 3056-3061.

[20] M. Merlino, M. Boiani, H. Cerecetto and M. González, "2-Benzyl-2-Methyl-2H-Benzimidazole 1,3-Dioxide DerivaTives: Spectroscopic And Theoretical Study," Spectrochimica Acta Part A, Vol. 67, No. 2, 2007, pp. 540-549. doi:10.1016/j.saa.2006.08.013

[21] M. O. Daodu and O. J. Babayemi, "Utilization of Some Edge-Row Plants as Forage in Nigeria," Pakistan Journal of Nutrition, Vol. 8, No. 8, 2009, pp. 1269-1274. doi:10.3923/pjn.2009.1269.1274 
[22] Wavefunction, Inc., "Spartan'04 for Windows," Irvine, California, USA.

[23] D. E. Wachenheim, L. L. Blythe and A. M. Craig, "Characterization of Rumen Bacterial Pyrrolizidine Alkaloid Biotransformation in Ruminants of Various Species," Veterinary \& Human Toxicology, Vol. 34, No. 6, 1992, pp. 513-517.

[24] H. Korbekandi, P. Mather, J. Gardiner and G. Stephens, "Reduction of Aliphatic Nitro Groups Using an Obligately Anaerobic Whole Cell Biocatalyst," Enzyme and Microbial Technology, Vol. 42, No. 4, 2008, pp. 308-314. doi:10.1016/j.enzmictec.2007.10.009

[25] A. O. Pacheco, E. Kagohara, L. H. Andrade, J. V. Comasseto, I. H.-S. Crusius, C. R. Paula and A. L. M. Porto,
"Biotransformations of Nitro-Aromatic Compounds to Amines and Acetamides by Tuberous Roots of Arracacia Xanthorrhiza and Beta Vulgaris and Associated Microorganism (Candida guilliermondii)," Enzyme and Microbial Technology, Vol. 42, No. 1, 2007, pp. 65-69. doi:10.1016/j.enzmictec.2007.08.001

[26] J. W. Czerkawski and G. Breckenridge, "Design and Development of a Long-Term Rumen Simulation Technique (Rusitec)," British Journal of Nutrition, Vol. 38, No. 3, 1977, pp. 371-384. doi:10.1079/BJN19770102

[27] J. B. Russell and H. J. Strobel, "Microbial Energetics. Quantitative Aspects of Ruminant Digestion and Metabolism," CABI, Wallingford, 2005, pp. 229-261. 